\title{
Energy function, formation rate and low-metallicity environment of fast radio bursts
}

\author{
G. Q. Zhang, ${ }^{1}$ F. Y. Wang, ${ }^{1,2 \star}$ \\ ${ }^{1}$ School of Astronomy and Space Science, Nanjing University, Nanjing 210093, China \\ ${ }^{2}$ Key Laboratory of Modern Astronomy and Astrophysics (Nanjing University), Ministry of Education, Nanjing 210093, China
}

5 June 2019

\begin{abstract}
In this paper, we investigate the energy function, formation rate and environment of fast radio bursts (FRBs) using Parkes sample and Australian Square Kilometer Array Pathfinder (ASKAP) sample. For the first time, the metallicity effect on the formation rate is considered. If FRBs are produced by the mergers of compact binaries, the formation rate of FRBs should have a time delay relative to cosmic star formation rate (CSFR). We get the time delay is about 3-5 Gyr and the index of differential energy function $\gamma\left(d N / d E \propto E^{-\gamma}\right)$ is between 1.6 and 2.0 from redshift cumulative distribution. The value of $\gamma$ is similar to that of FRB 121102, which indicates single bursts may share the same physical mechanism with the repeaters. In another case, if the formation rate of FRB is proportional to the SFR without time delay, the index $\gamma$ is about 2.3. In both cases, we find that FRBs may prefer to occur in low-metallicity environment with $12+\log (\mathrm{O} / \mathrm{H}) \simeq 8.40$, which is similar to those of long gamma-ray bursts (GRBs) and hydrogen-poor superluminous supernovae (SLSNe-I).
\end{abstract}

Key words: radio continuum - general - stars: neutron

\section{INTRODUCTION}

Fast radio bursts (FRBs) with anomalously high dispersion measure (DM) (Lorimer et al. 2007; Thornton et al. 2013; Petroff et al. 2015; Katz 2018; Platts et al. 2018) are mysterious radio transients, and have been detected at frequencies between $400 \mathrm{MHz}$ and $8 \mathrm{GHz}$ by ground-based radio telescopes. By now, more than sixty FRBs have been discovered, only FRB 121102 and FRB 180814 are repeating (Spitler et al. 2016; Scholz et al. 2016; CHIME/FRB Collaboration et al. 2019). The localization of the FRB 121102 (Chatterjee et al. 2017; Marcote et al. 2017; Tendulkar et al. 2017) confirmed the cosmological origin of this source (at $z=0.19$ ). If many redshifts of FRBs are measured by upcoming instruments, the combined redshift and DM can be used as cosmological purpose, including measuring the baryon number density (Deng \& Zhang 2014; Keane et al. 2016), measuring cosmic proper distance (Yu \& Wang 2017), constraining the cosmological parameters (Zhou et al. 2014; Gao et al. 2014; Walters et al. 2018), measuring the Hubble Constant and cosmic curvature if some repeating FRBs are gravitationally lensed ( $\mathrm{Li}$ et al. 2018), probing compact dark matter through strong lensed FRBs (Muñoz et al. 2016; Wang \& Wang 2018) and testing

* E-mail: fayinwang@nju.edu.cn
Einstein's Weak Equivalence Principle (WEP) (Wei et al. 2015; Yu et al. 2018).

Because physical constraints on the progenitors of FRBs are few, there are many theoretical models. They generally fall into two categories: emission from compact binary merger (Kashiyama et al. 2013; Totani 2013; Wang et al. 2016; Zhang 2016; Liu et al. 2016; Yamasaki et al. 2018) and emission from a neutron star (Popov \& Postnov 2010; Falcke \& Rezzolla 2014; Zhang 2014; Kulkarni et al. 2014; Dai et al. 2016; Katz 2016; Cordes \& Wasserman 2016; Metzger et al. 2017). Some progenitor models (Zhang 2014; Connor et al. 2016; Cordes \& Wasserman 2016; Metzger et al. 2017) connect FRBs and young neutron stars produced from supernovae or gamma-ray bursts (GRBs), so that their formation rate may track the cosmic star formation rate (CSFR). During the past years, some statistical studies of FRBs have been performed to constrain the models (Bera et al. 2016; Caleb et al. 2016; Katz 2016; Oppermann et al. 2016; Lu \& Kumar 2016; Vedantham et al. 2016; Wang \& Yu 2017; Fialkov \& Loeb 2017; Lawrence et al. 2017; Cao et al. 2017, 2018; Macquart \& Ekers 2018; Lu \& Piro 2019; Zhang et al. 2019; Wang \& Zhang 2019).

The energy function, formation rate and burst environment are crucial constraints on the progenitor models. For example, the host galaxy of FRB 121102 is found to be a low-metallicity, star-forming dwarf galaxy 
(Tendulkar et al. 2017). More recently, FRB 171020 with the smallest recorded $\mathrm{DM}=114 \mathrm{pc} \mathrm{cm}^{-3}$ discovered by Australian Square Kilometer Array Pathfinder (ASKAP) survey is possible associated with the Sc galaxy ESO 601G036 (Mahony et al. 2018). This galaxy is also metal-poor, and shares similar properties with the galaxy hosting the repeating FRB 121102. However, whether the two galaxies represent the broader FRB population property is unknown. Meanwhile, observations show that both long GRBs and hydrogen-poor superluminous supernovae (SLSNe-I) exhibit a strong preference for low-mass, low-metallicity galaxies (Fruchter et al. 2006; Wang \& Dai 2014; Lunnan et al. 2014). Here we provide a detailed study on the energy function, rate and environment of FRBs using the Parkes sample and ASKAP sample.

This paper is organized as follows. In section 2, we present FRB samples and derive the pseudo redshifts for FRBs. In section 3, considering time delay and the metallicity of host galaxy, we fit the cumulative distribution with two FRB rate models. Finally, summary is given in section 4. In this paper, we adopt the $\Lambda$ CDM model with $H_{0}=67.74 \mathrm{~km} \mathrm{~s}^{-1} \mathrm{Mpc}^{-1}, \Omega_{b}=0.31$ and $\Omega_{\Lambda}=0.69$ (Planck Collaboration et al. 2016).

\section{FRB SAMPLES}

We select the data from http://www.frbcat.org, which includes FRBs observed by Parkes, UTMOST, ASKAP and Arecibo, etc (Petroff et al. 2016). These telescopes have different central frequencies, bandwidths and thresholds. So it's unreasonable to put them together to investigate statistical nature of FRBs. We only consider FRBs observed by the same telescope. We select the FRBs observed by Parkes and ASKAP. The Parkes sample contains 28 FRBs, while the ASKAP sample has 23 FRBs.

The DM of FRB can be divided into several parts

$\mathrm{DM}=\mathrm{DM}_{\mathrm{MW}}+\mathrm{DM}_{\mathrm{IGM}}+\frac{\mathrm{DM}_{\text {host }}+\mathrm{DM}_{\mathrm{src}}}{1+\mathrm{z}}$,

where $\mathrm{DM}_{\mathrm{MW}}, \mathrm{DM}_{\mathrm{IGM}}, \mathrm{DM}_{\text {host }}$ and $\mathrm{DM}_{\mathrm{src}}$ are the contributions from the Milky Way, the intergalactic medium (IGM), the host galaxy and the source. According the position of $\mathrm{FRB}$, the $\mathrm{DM}_{\mathrm{MW}}$ can be inferred from the galactic distribution of free electrons. The values of $\mathrm{DM}_{\mathrm{MW}}$ have been given in the FRB catalog, which are based on the NE2001 model (Cordes \& Lazio 2002). The $\mathrm{DM}_{\mathrm{IGM}}$ can be calculated as (Inoue 2003; Ioka 2003; Deng \& Zhang 2014)

$\operatorname{DM}_{\mathrm{IGM}}(z)=\frac{3 c H_{0} \Omega_{b}}{8 \pi G m_{p}} f_{\mathrm{IGM}} \int_{0}^{z} \frac{H_{0} f_{e}\left(z^{\prime}\right)\left(1+z^{\prime}\right)}{H\left(z^{\prime}\right)} d z^{\prime}$,

where $f_{\text {IGM }} \sim 0.83$ is the fraction of baryon mass in the IGM (Fukugita et al. 1998) and $f_{e}(z)$ is the number ratio between free electrons and baryons in IGM. $f_{e}(z)$ can be calculated as

$f_{e}(z) \simeq \frac{3}{4} \chi_{e, H}(z)+\frac{1}{8} \chi_{e, H e}(z)$

where $\chi_{e, H}(z)$ and $\chi_{e, H e}(z)$ are the cosmic ionization of hydrogen and helium, respectively. Because the redshifts of FRBs are small, we can ignore the evolution of these parameters and take $\chi_{e, H}(z) \simeq 1, \chi_{e, H e}(z) \simeq 1$. Finally, $f_{e}(z)=7 / 8$ is chosen in our calculation.
However, the values of $\mathrm{DM}_{\text {host }}$ and $\mathrm{DM}_{\mathrm{src}}$ are highly uncertain. The only verified host galaxy of FRB 121102 shows $55 \mathrm{pccm}^{-3} \leq \mathrm{DM}_{\text {host }}+\mathrm{DM}_{\mathrm{src}} \leq 225 \mathrm{pc}$ $\mathrm{cm}^{-3}$ (Tendulkar et al. 2017). However, whether the properties of the host galaxies of repeating FRBs and nonrepeating FRBs are similar is still in debate. In this paper, we adopt the distribution of DM given by Yang et al. (2017). They use $21 \mathrm{FRBs}$ to infer $\mathrm{DM}_{\text {host }}+\mathrm{DM}_{\mathrm{src}}=$ $267.00_{-110.68}^{+172.53} \mathrm{pc} \mathrm{cm}^{-3}$. We simulate 20000 points and use these points to obtain the pseudo redshifts through equations (1) and (2). It must be noted that there could be a correlation between the host DM and scattering, free-free absorption and other properties, which may also affect the pseudo redshifts.

\section{METHOD AND RESULTS}

\subsection{Cumulative redshift distribution}

Because the number of FRBs is small, it's preferable to use the cumulative distribution rather than differential distribution. It can avoid binning of the data. The width of binning has significant impact on the results when the number of events is not enough. According the pseudo redshifts given by equation (2), we derive the cumulative redshift distributions for Parkes sample and ASKAP sample, which are shown in Figure 1 and Figure 2, respectively. The blue line is the cumulative distribution and the shadow region is the uncertainties of the redshifts, which are caused by the uncertainty of $\mathrm{DM}_{\text {host }}+\mathrm{DM}_{\text {src }}$. It's obvious that the uncertainty of $\mathrm{DM}_{\text {host }}+\mathrm{DM}_{\text {src }}$ can slightly affect the cumulative distribution.

We use two models to fit the cumulative redshift distributions. If FRBs origin from mergers of compact binaries, the formation rate should be proportional to the rate of compact binaries and has a time delay relative to CSFR. We use $\dot{R}_{m}(z, \tau)$ as the rate of the compact binaries, where $\tau$ is the time delay. Besides, according to the observation (Tendulkar et al. 2017), the metallicity of the host galaxy of FRB 121102 is poor. Thus we speculate that FRBs occur in low-metallicity environment. The metallicity evolution $\Psi(Z, z)$ is considered. Therefore, the formation rate of FRB can be calculated as

$\rho_{F R B}(z) \propto \dot{R}_{m}(z, \tau) \Psi(Z, z)$.

The second model is based on the progenitors model of FRBs associated with young neutron stars produced from supernovae or GRBs. In this case, the effect of time delay can be ignored and the formation rate is

$\rho_{F R B}(z) \propto \rho(z) \Psi(Z, z)$,

where $\rho(z)$ is the CSFR.

In order to derive the theoretical cumulative redshift distribution, the thresholds of telescopes must be considered. Assuming the energy distribution of FRBs satisfies the differential energy distribution $\Phi(E)=d N / d E \propto E^{-\gamma}$, the theoretical cumulative redshift distribution can be obtained through

$N(<z)=A \int_{0}^{z} \rho_{F R B}(z)\left[\int_{0}^{1} \eta(\varepsilon) \int_{E_{t h} / \varepsilon}^{E_{\max }} \Phi(E) d E d \varepsilon\right] \frac{d V(z)}{1+z}$, 
where $A$ is the normalized constant, $\varepsilon$ is the beam efficiency, $\eta(\varepsilon)$ is the distribution of $\varepsilon, E_{t h}$ is the minimum energy which can be observed by telescopes and $E_{\max }$ is the maximum energy of FRBs. Below, we will discuss time delay, metallicity, beam efficiency and threshold in details.

\subsection{CSFR and Time Delay}

We adopt the CSFR given by Madau \& Dickinson (2014)

$\rho(z)=0.015 \frac{(1+z)^{2.7}}{1+[(1+z) / 2.9]^{5.6}} \mathrm{M}_{\odot} \operatorname{year}^{-1} \mathrm{Mpc}^{-3}$.

If FRBs are produced by mergers of compact binaries, the formation rate should have a time delay to the CSFR. We take the time delay $\tau$ as $t(z)-t\left(z^{\prime}\right)$, where $z$ is the redshift when the compact binaries were formed, $z^{\prime}$ is the redshift when the FRBs occurred and $t$ is the universe age at redshift $z$. The time delay $\tau$ should be determined by the time scale of the inspiral and formation process of compact binaries. There are many works to discuss the model of $\tau$. In our analysis, we take the probability distribution of $\tau$ as $P(\tau)$ and an empirical expression of $P(\tau)$ is taken as (Cao et al. 2018)

$P(\tau) \propto\left(\frac{\tau}{\tau_{c}}\right)^{-1} e^{-\tau_{c} / \tau}$,

where $\tau_{c}$ is a typical time scale. Considering the time delay, the rate of the compact binaries merger can be derived by

$\dot{R}_{m}(z) \propto \int_{0}^{z_{\max }} \rho\left(z^{\prime}\right) P\left(t(z)-t\left(z^{\prime}\right)\right) \frac{d t}{d z^{\prime}} d z^{\prime}$,

where $d t / d z=-[(1+z) H(z)]^{-1}$ and $z_{\max }$ is the maximum redshift when first stars formed.

\subsection{Beam Efficiency}

The positions of FRBs within the receiver beam of telescope have strong impact on the observed fluence. Therefore, for a multi-beam receiver, it is important to consider the beam efficiency. The Airy disk is adopted to illustrate the beam efficiency (Bera et al. 2016; Vedantham et al. 2016),

$\varepsilon=\left[\frac{2 J_{1}(a)}{a}\right]^{2}$,

where $J_{1}(a)$ is the first order Bessel function and $a=r / r_{c}$ represents the offset to the center of the main beam. The typical value of $\varepsilon$ is $\varepsilon(a=0)=1$, and $\varepsilon(a=3.83)=0$. Considering the beam efficiency, the observed fluence is

$F_{o b s}=\varepsilon F_{e f f}$,

where $F_{\text {eff }}$ is the effective fluence which removes the beam effect. We also consider the probability distribution of $\varepsilon$ given by Niino (2018)

$\eta(\varepsilon)=\frac{2}{a_{\max }^{2}} a(\varepsilon)\left|\frac{d a}{d \varepsilon}\right|$,

where $a_{\max }$ is the maximum value of $a$ that satisfies $\varepsilon\left(a_{\max }\right)=0, a(\varepsilon)$ is the inverse function and $d a / d \varepsilon$ is the differential function of $a(\varepsilon)$.

We adopt the Airy disk to describe the beam efficiency of Parkes. This is a simplification and the efficiency of radio receiver also depend on the frequency. Thus, the spectra of FRBs may affect the efficiency. In our analysis, a power-law spectrum is adopted for all FRBs. In each sample, all FRBs have the same spectrum, power-law index, central frequency and bandwidth. Therefore, the dependence can be ignored.

As for the beam efficiency of ASKAP, due to its fly'seye configuration, it's unacceptable to use a single airy disk to describe its beam efficiency. James et al. (2019) derived the best and worst beam efficiencies for closepack36 and square $6 \times 6$ configurations. Because the contribution from closepack36 configuration is much larger than that of square $6 \times 6$, we take the best beam efficiency from closepack36 in our analysis.

\subsection{Threshold}

The thresholds of telescopes play an important role on the observed cumulative redshift distribution of FRBs. As for the Parkes, the fluence sensitivity is adopted as (Bera et al. 2016)

$F_{\nu, t h}=0.04 \frac{S / N}{\varepsilon} \sqrt{\frac{\omega}{1 \mathrm{~ms}}} \mathrm{Jy} \mathrm{ms}$,

where $S / N$ is the signal-to-noise, and we take it as 10. $\omega$ is the typical duration of FRBs, which can be calculated from

$\omega=\sqrt{\omega_{\mathrm{in}}^{2}(1+z)^{2}+\omega_{\mathrm{DM}}^{2}+\omega_{\mathrm{sc}}^{2}}$,

where $\omega_{\text {in }}$ is the intrinsic width, $\omega_{\mathrm{DM}}$ is the width caused by residual dispersion across a single frequency channel and $\omega_{\text {sc }}$ is the contribution by the scattering. A typical intrinsic width $\omega_{\text {in }} \simeq 1.3 \mathrm{~ms}$ is adopted. Assuming $\Delta \nu_{c} / \nu_{0} \ll 1$, the $\omega_{\text {DM }}$ can be calculated as (Bera et al. 2016)

$\omega_{\mathrm{DM}} \simeq 8.3 \times 10^{6} \frac{\mathrm{DM}(\mathrm{z}) \Delta \nu_{\mathrm{c}}}{\nu_{0}^{3}}$,

where $\Delta \nu_{c}$ is the single frequency channel bandwidth and $\nu_{0}$ is the central frequency. As for the $\omega_{\mathrm{sc}}$, Macquart \& Koay (2013) gave its form

$\omega_{\mathrm{sc}}=\frac{k_{s c}[1-\sqrt{z /(1+z)}]}{\nu_{0}^{4}(1+z)} \times \int_{0}^{z} \frac{H_{0}}{H\left(z^{\prime}\right)} d z^{\prime} \int_{0}^{z} \frac{H_{0}\left(1+z^{\prime}\right)^{3}}{H\left(z^{\prime}\right)} d z^{\prime}$

with $k_{\mathrm{sc}}=4.2 \times 10^{13} \mathrm{~ms} \mathrm{MHz}^{4}$. According to eqs. (13) (16), the threshold of Parkes can be obtained.

For the ASKAP, we adopt $26 \mathrm{Jy} \mathrm{ms}$ as its threshold, which corresponds the signal-to-noise of 9.5 (Shannon et al. 2018). We don't consider the threshold evolution with redshift for ASKAP. It should be noted that the threshold of ASKAP is much larger than that of Parkes. So the redshifts of FRBs observed by ASKAP are small. Actually, The maximum pseudo redshift of FRBs observed by ASKAP is only 1.006. Thus we can ignore the threshold evolution.

We assume that the differential energy distribution of FRBs satisfies a power-law form

$\Phi(E)=\frac{d N}{d E} \propto E^{-\gamma}$.

In this equation, the energy $E$ is not the intrinsic energy but the effective energy. Only the beam effect is considered in the effective energy, other selection effects such as propagation effect are not considered. These effects are complicated, and we have little understand of these. Considering 
the threshold of the telescopes, only FRBs with large energy can be observed. The minimum energy that can be observed at redshift $z$ is

$E_{t h}=4 \pi d_{c}(z)^{2}(1+z) \Delta \nu F_{\nu, t h} k(z)$,

where $d_{c}$ is the comoving distance and $k(z)$ is the factor of the K-correction. The spectrum of FRB is still unclear. For simplicity, we assume that FRBs satisfy a power-law spectrum, $F_{\nu} \propto \nu^{-\beta}$. Then $k(z)$ is

$k(z)=\frac{\nu_{\max }^{1-\beta}-\nu_{\min }^{1-\beta}}{\left[(1+z) v_{2}\right]^{1-\beta}-\left[(1+z) v_{1}\right]^{1-\beta}}$,

where $\nu_{\max }$ and $\nu_{\min }$ are the minimum frequency and maximum frequency at rest frame, respectively. As for Parkes, $\nu_{2}$ and $\nu_{1}$ are taken as $1522 \mathrm{MHz}$ and $1182 \mathrm{MHz}$. The index of spectrum is uncertain. Previous studies have shown the index is $\beta=0.3 \pm 0.9$ for FRB 131104 (Ravi et al. 2015) and $\beta=1.3 \pm 0.5$ for FRB 150418 (Keane et al. 2016). Therefore, we take $\beta=0.8$ for Parkes sample. The $\nu_{2}$ and $\nu_{1}$ is $1465 \mathrm{MHz}$ and $1129 \mathrm{MHz}$ for ASKAP. Shannon et al. (2018) obtained that the index of spectrum is $1.8 \pm 0.3$ for ASKAP data. We also use this index for ASKAP. Assuming all FRBs redshifts are in the range of $0-4$, we obtain $\nu_{\max }=7610$ $\mathrm{MHz}$ and $\nu_{\min }=1129 \mathrm{MHz}$.

\subsection{Metallicity}

Another important effect is the metallicity of host galaxy. Tendulkar et al. (2017) found that the host galaxy of FRB 121102 is a low-metallicity galaxy. The $3 \sigma$ limit of its galaxy is $\log _{10}([\mathrm{O} / \mathrm{H}])+12<8.4$, which indicates that host galaxies of FRBs are more likely to be metal-poor galaxies. In order to describe the cosmic metallicity evolution, we employ the method developed by Langer \& Norman (2006). Panter et al. (2004) obtained the galaxy stellar mass function

$\psi(M)=A\left(\frac{M}{M_{\odot}}\right)^{\alpha} e^{-M / M_{\odot}}$,

where $\alpha=-1.16, A=7.8 \times 10^{-3} h^{3} \mathrm{Mpc}^{-3}$ and $M_{\odot}$ is the solar mass. Through this equation, the fraction of galaxies with mass less than $M$ can be calculated as

$\Psi(M)=\frac{\int_{0}^{M} M \psi(M) d M}{\int_{0}^{\infty} M \psi(M) d M}=\frac{\widehat{\Gamma}\left(\alpha+2, M / M_{\odot}\right)}{\Gamma(\alpha+2)}$,

where $\Gamma$ and $\widehat{\Gamma}$ is the complete and incomplete gamma function. Considering the mass-metallicity relation $M / M_{\odot}=$ $\left(Z / Z_{\odot}\right)^{\zeta}$ (Savaglio et al. 2005) at $z=0.7$ and the evolution $Z=Z_{\odot} 10^{-0.15 z}$ (Langer \& Norman 2006), the $\frac{M}{M_{\odot}}$ can be transformed into $\frac{Z}{Z_{\odot}}$ :

$\Psi(Z, z)=\frac{\widehat{\Gamma}\left[\alpha+2,\left(Z / Z_{\odot}\right)^{\zeta} 10^{0.15 \zeta z}\right]}{\Gamma(\alpha+2)}$,

where $\zeta=2$ and $Z_{\odot}$ is the solar metallicity. This function describes the fraction mass density belonging to metallicities below metallicity $Z$ at redshift $z$ and has been used to investigate the CSFR and the gamma-ray bursts (Li 2008; Wang \& Dai 2009; Salvaterra et al. 2012; Wang 2013). The above equation is based on the galaxy mass function, massmetallicity relation and metallicity evolution with redshift. These equations have some simplifications and the validity of these simplifications need to be examined. Li (2008) studied these simplifications in detail and found it's enough to adopt this analytical form of metallicity evolution (Hao \& Yuan 2013). Thus we use equation (22) and ignore its uncertainty.

\subsection{Results}

We use Markov Chain Monte Carlo (MCMC) method to derive the best-fitting parameters. The time delay $\tau$, the power-law index of energy function $\gamma$ and the metallicity $Z$ are taken as free parameters. According to the Bayes' theorem, the posterior probability can be derived through

$p(\theta \mid d)=\frac{p(d \mid \theta) p(\theta)}{p(d)}$,

where $\theta$ is the free parameters and $d$ is the data of FRBs. We adopt the uniform distribution as the prior probability. In order to get the goodness of fit, we adopt the KolmogorovSmirnov test. The p-value of this test is considered as the likelihood. Through this likelihood, we derive the posterior probability for all cases. Considering the time delay, we obtain $\tau=2.77_{-1.90}^{+2.86}$ Gyr, $\gamma=1.63_{-0.25}^{+0.32}, Z=0.46_{-0.31}^{+0.35} Z_{\odot}$ for Parkes sample and $\tau=5.50_{-3.62}^{+3.01} \mathrm{Gyr}, \gamma=2.07_{-0.14}^{+0.14}$, $Z=0.52_{-0.34}^{+0.32} Z_{\odot}$ for ASKAP sample. The results are shown in figures 1 and 2 , respectively. In these figures, the blue line is observed distribution of FRBs and shadow region is the uncertainties of the redshifts. The red line is the bestfitting result. If the time delay is not considered, we find $\gamma=2.37_{-0.16}^{+0.12}, Z=0.52_{-0.34}^{+0.34} Z_{\odot}$ for Parkes sample and $\gamma=2.40_{-0.08}^{+0.08}, Z=0.52_{-0.34}^{+0.32} Z_{\odot}$ for ASKAP sample. The results are shown in figures 3 and 4 . We also list the bestfitting parameters in table 1.

The time delay derived from Parkes sample is $2.77_{-1.90}^{+2.86}$ Gyr, which is similar to that for ASKAP sample $\tau=$ $5.50_{-3.62}^{+3.01} \mathrm{Gyr}$ at $1 \sigma$ level. The large error of time delay is caused by the scarcity of the FRBs. Cao et al. (2018) also consider the time delay, but they obtained the time delay is about 350 Myr, which is much smaller than ours. This inconsistency is due to the fact that they do not consider the metallicity effect.

Another important result is the index of the differential energy distribution. Considering the time delay, the index for Parkes sample is $\gamma=1.63_{-0.25}^{+0.32}$, which is similar to the result of Cao et al. (2018). Besides, Wang \& Yu (2017) and Law et al. (2017) obtained that the index of the differential energy distribution for FRB 121102 is also about 1.7. However, Gourdji et al. (2019) found the value of $\gamma=2.8 \pm 0.3$ for FRB 121102 using a sample of lowenergy bursts. Wang \& Zhang (2019) found a universal energy distribution with $\gamma \sim 1.7$ for FRB 121102 using bursts observed by different telescopes. As for ASKAP sample, the index is $2.07_{-0.14}^{+0.14}$. The difference between these two samples is caused by the selection effect. The threshold of ASKAP is much larger than that of Parkes, which causes the mean energy of ASKAP sample is larger. Lu \& Kumar (2016) proposed that the index of differential energy distribution is $1.5 \leq \gamma \leq 2.2$ for the repeating FRB 121102. This value is consistent with our result. If the time delay is not considered, the indices are $2.37_{-0.16}^{+0.12}$ and $2.40_{-0.08}^{+0.08}$ for Parkes and ASKAP samples, respectively.

We find that the metallicity is low for all the cases. Through $[12+\log (\mathrm{O} / \mathrm{H})]_{\mathrm{FRB}}=[12+\log (\mathrm{O} / \mathrm{H})]_{\text {sun }}+$ 
$\log \left(\mathrm{Z} / \mathrm{Z}_{\odot}\right)$, the metallicity $[12+\log (\mathrm{O} / \mathrm{H})]$ are $8.35_{-0.50}^{+0.25}$, $8.40_{-0.47}^{+0.21}, 8.41_{-0.47}^{+0.22}, 8.38_{-0.51}^{+0.24}$ for Parkes sample with time delay, ASKAP sample with time delay, Parkes sample without time delay, ASKAP sample without time delay, respectively. We take $[12+\log (\mathrm{O} / \mathrm{H})]_{\text {sun }}=8.69$ (Asplund et al. 2009) as the solar metallicity. Although the time delay and energy distribution are different in the two models, the metallicities are similar. According to the observations, the host galaxies of FRB 121102 (Tendulkar et al. 2017) have low metallicity. Although whether the environment of repeating FRBs and non-repeating FRBs is the same is still unknown, our conclusion is consistent with the observation for repeating FRB 121102. Considering the large uncertainty, our results only provide a trend that the FRBs are more likely to occur in low-metallicity environment. The larger error of metallicity is mainly due to the small sample of FRBs. As more and more FRBs are observed, we expect more precise constraint on metallicity.

Besides, observations confirmed that long GRBs and SLSNe-I prefer to occur in low-metallicity host galaxies (Fruchter et al. 2006; Lunnan et al. 2014). The similar properties of host galaxy may indicate that they have similar progenitor model.

\section{SUMMARY AND DISCUSSION}

In this paper, we collect the FRBs observed by Parkes and ASKAP. We derive the pseudo redshifts from their DMs and obtain the cumulative distribution for each sample. Considering the effects of time delay and the metallicity, we construct the model of theoretical cumulative redshift distribution and obtain the best fit through MCMC method.

We find the time delay is $2.77_{-1.90}^{+2.86} \mathrm{Gyr}$ and $5.50_{-3.62}^{+3.01}$ Gyr for Parkes sample and ASKAP sample, respectively. This time delay is very large and is consistent with the time delay for GRBs (Wanderman \& Piran 2015). The most important result is that FRBs prefer to occur in low-metallicity environment for all the cases for the first time. The cut-off metallicity is about $[12+\log (\mathrm{O} / \mathrm{H})]=8.30$. These results suggest that FRBs occur in low-metallicity environment, which is similar as those of GRBs and SLSNe-I.

In our analysis, we use a simplified model to describe cosmic metallicity evolution and assume the metallicity is independent of the CSFR. However, there are some studies indicating that metallicity is connected with galaxy stellar mass and star formation rate in galaxy (Mannucci et al. 2010). If this relation is rubust, the star formation rate has a strong effect on metallicity evolution, our assumptions may introduce bias. However, Sánchez et al. (2017) proposed that this relation is not strong enough. Besides, this relation is only applied to the star formation rate of a galaxy. In our calculation, we adopt the CSFR rather than the star formation rate of a particular galaxy. Therefore, this relation is ignored in our analysis.

As for the beam efficiency for ASKAP, we adopt the best efficiency for the closepack configuration given by James et al. (2019). In their analysis, they provide 4 beam efficiencies, the best and worst beam efficiency for closepack36 configuration and square $6 \times 6$ configuration. The square $6 \times 6$ configuration is ignored, because its contribution is very small. We use the worst beam efficiency for the closepack configuration in ASKAP without time delay model to test whether beam efficiency can significantly affect our results. Using the worst beam efficiency, we obtain $\gamma=2.40_{-0.08}^{+0.09}, Z=0.52_{-0.34}^{+0.32}$, which is consistent with the results for the best beam efficiency. This suggests the effect of different beam efficiencies can be ignored.

It must be noted that we adopt a single power law to describe the spectrum of FRBs. This may import some uncertainties. In order to test the influence of spectrum, we also take the power-law indices $\beta=0,1.8$ for Parkes without time delay. We derive $\gamma=2.49_{-0.13}^{+0.13}, Z=0.53_{-0.34}^{+0.32}$ for $\beta=0$ and $\gamma=2.24_{-0.18}^{+0.16}, Z=0.52_{-0.35}^{+0.33}$ for $\beta=1.8$. The best-fitting results for different $\beta$ are consistent with each other in $1 \sigma$ confidence level. Therefore, the uncertainty of spectral indices doesn't significantly affect our results. Recently, some observations found that the intrinsic spectrum of FRBs may be not a single power law (Hessels, et al. 2019). In this paper, we consider the spectra are power-law forms. If the spectra are not power laws, the above analysis should be reconsidered. In the future, the constraint on spectra will be more reliable. Our results can be tested with accurate spectrum.

\section{ACKNOWLEDGEMENTS}

We thank the anonymous referee for useful suggestions which were helpful for improving the manuscript. We also thank H. Qiu for helpful discussion on the beam efficiency for ASKAP. This work is supported by the National Natural Science Foundation of China (grant U1831207).

\section{REFERENCES}

Asplund M., Grevesse N., Sauval A. J., Scott P., 2009, ARA\&A, 47,481

Bera A., Bhattacharyya S., Bharadwaj S., Bhat N. D. R., Chengalur J. N., 2016, MNRAS, 457, 2530

CHIME/FRB Collaboration et al., 2019, Nature, 566, 235

Caleb M., Flynn C., Bailes M., Barr E. D., Hunstead R. W., Keane E. F., Ravi V., van Straten W., 2016, MNRAS, 458,708

Cao X.-F., Xiao M., Xiao F., 2017, RAA, 17, 14

Cao X.-F., Yu Y.-W., Zhou X., 2018, ApJ, 858, 89

Chatterjee S., et al., 2017, Nature, 541, 58

Connor L., Sievers J., Pen U.-L., 2016, MNRAS, 458, L19

Cordes J. M., Lazio T. J. W., 2002, arXiv e-prints, astro-ph/0207156

Cordes J. M., Wasserman I., 2016, MNRAS, 457, 232

Dai, Z. G., Wang, J. S., Wu, X. F., \& Huang, Y. F. 2016, ApJ, 829,27

Deng W., Zhang B., 2014, ApJ, 783, L35

Falcke H., Rezzolla L., 2014, A\&A, 562, A137

Fialkov A., Loeb A., 2017, ApJ, 846, L27

Fruchter A. S., et al., 2006, Nature, 441, 463

Fukugita M., Hogan C. J., Peebles P. J. E., 1998, ApJ, 503, 518

Gao H., Li Z., Zhang B., 2014, ApJ, 788, 189

Gourdji K., Michilli D., Spitler L. G., Hessels J. W. T., Seymour A., Cordes J. M., Chatterjee S., 2019, arXiv e-prints, arXiv:1903.02249

Hao J.-M., Yuan Y.-F., 2013, ApJ, 772, 42

Hessels J. W. T., et al., 2019, ApJ, 876, L23

Inoue A. K., 2003, PASJ, 55, 901

Ioka K., 2003, ApJ, 598, L79 
James C. W., et al., 2019, Publ. Astron. Soc. Australia, 36, e009 Kashiyama K., Ioka K., Mészáros P., 2013, ApJ, 776, L39

Katz J. I., 2016, ApJ, 826, 226

Katz J. I., 2018, Progress in Particle and Nuclear Physics, 103, 1

Keane E. F., et al., 2016, Nature, 530, 453

Kulkarni S. R., Ofek E. O., Neill J. D., Zheng Z., Juric M., 2014, ApJ, 797, 70

Langer N., Norman C. A., 2006, ApJ, 638, L63

Law C. J., et al., 2017, ApJ, 850, 76

Lawrence E., Vander Wiel S., Law C., Burke Spolaor S., Bower G. C., 2017, AJ, 154, 117

Li L.-X., 2008, MNRAS, 388, 1487

Li Z.-X., Gao H., Ding X.-H., Wang G.-J., Zhang B., 2018, Nature Communications, 9, 3833

Liu T., et al., 2016, ApJ, 826, 82

Lorimer D. R., Bailes M., McLaughlin M. A., Narkevic D. J., Crawford F., 2007, Science, 318, 777

Lu W., Kumar P., 2016, MNRAS, 461, L122

Lu W., Piro A. L., 2019, arXiv e-prints, arXiv:1903.00014

Lunnan R., et al., 2014, ApJ, 787, 138

Macquart J. P., Ekers R. D., 2018, MNRAS, 474, 1900

Macquart J.-P., Koay J. Y., 2013, ApJ, 776, 125

Madau P., Dickinson M., 2014, ARA\&A, 52, 415

Mahony E. K., et al., 2018, ApJ, 867, L10

Mannucci F., Cresci G., Maiolino R., Marconi A., Gnerucci A., 2010, MNRAS, 408, 2115

Marcote B., et al., 2017, ApJ, 834, L8

Metzger B. D., Berger E., Margalit B., 2017, ApJ, 841, 14

Muñoz J. B., Kovetz E. D., Dai L., Kamionkowski M., 2016, Phys. Rev. Lett., 117, 091301

Niino Y., 2018, ApJ, 858, 4

Oppermann N., Connor L. D., Pen U.-L., 2016, MNRAS, 461, 984

Panter B., Heavens A. F., Jimenez R., 2004, MNRAS, 355, 764

Petroff E., et al., 2015, MNRAS, 447, 246

Petroff E., et al., 2016, Publ. Astron. Soc. Australia, 33, e045

Planck Collaboration et al., 2016, A\&A, 594, A13

Platts E., Weltman A., Walters A., Tendulkar S. P., Gordin J. E. B., Kandhai S., 2018, arXiv e-prints, arXiv:1810.05836

Popov S. B., Postnov K. A., 2010, vaoa.conf, 129, vaoa.conf

Ravi V., Shannon R. M., Jameson A., 2015, ApJ, 799, L5

Salvaterra R., et al., 2012, ApJ, 749, 68

Sánchez S. F., et al., 2017, MNRAS, 469, 2121

Savaglio S., et al., 2005, ApJ, 635, 260

Scholz P., et al., 2016, ApJ, 833, 177

Shannon R. M., et al., 2018, Nature, 562, 386

Spitler L. G., et al., 2016, Nature, 531, 202

Tendulkar S. P., et al., 2017, ApJ, 834, L7

Thornton D., et al., 2013, Science, 341, 53

Totani T., 2013, PASJ, 65, L12

Vedantham H. K., Ravi V., Hallinan G., Shannon R. M., 2016, ApJ, 830, 75

Walters A., Weltman A., Gaensler B. M., Ma Y.-Z., Witzemann A., 2018, ApJ, 856, 65

Wanderman D., Piran T., 2015, MNRAS, 448, 3026

Wang F. Y., 2013, A\&A, 556, A90

Wang F. Y., Dai Z. G., 2009, MNRAS, 400, L10

Wang F. Y., Dai Z. G., 2014, ApJS, 213, 15

Wang Y. K., Wang F. Y., 2018, A\&A, 614, A50

Wang F. Y., Yu H., 2017, J. Cosmology Astropart. Phys., 03, 023

Wang F. Y., Zhang G. Q., 2019, arXiv e-prints, arXiv:1904.12408

Wang J.-S., Yang Y.-P., Wu X.-F., Dai Z.-G., Wang F.-Y., 2016, ApJ, 822, L7

Wei J.-J., Gao H., Wu X.-F., Mészáros P., 2015, Phys. Rev. Lett., 115,261101

Yamasaki S., Totani T., Kiuchi K., 2018, PASJ, 70, 39

Yang Y.-P., Luo R., Li Z., Zhang B., 2017, ApJ, 839, L25

Yu H., Wang F. Y., 2017, A\&A, 606, A3

Yu H., Xi S.-Q., Wang F.-Y., 2018, ApJ, 860, 173
Zhang B., 2014, ApJ, 780, L21

Zhang B., 2016, ApJ, 827, L31

Zhang G. Q., Wang F. Y., Dai Z. G., 2019, arXiv e-prints, arXiv: 1903.11895

Zhou B., Li X., Wang T., Fan Y.-Z., Wei D.-M., 2014, Phys. Rev. D, 89, 107303 


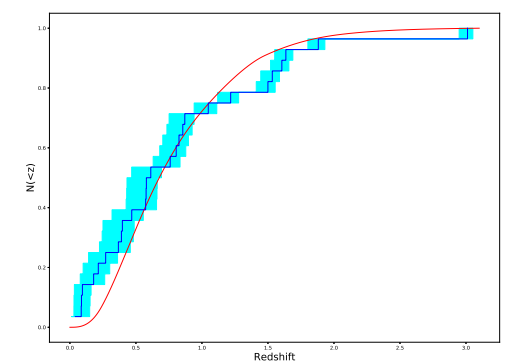

Figure 1. The cumulative distribution of 28 Parkes FRBs. The blue line is the observed distribution of FRBs and the shadow region is the uncertainties of the redshifts. The red line is the best-fitting result with $\tau=2.77 \mathrm{Gyr}, \gamma=1.63$ and $Z=0.46 Z_{\odot}$.

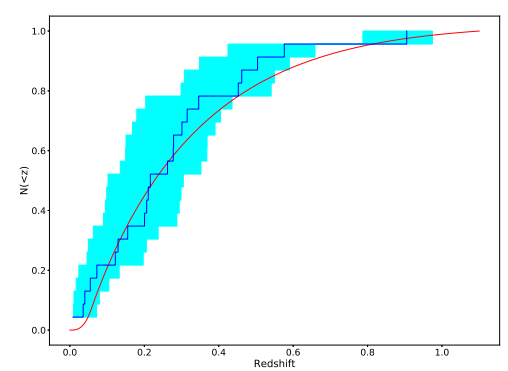

Figure 2. The cumulative distribution of 23 FRBs observed by ASKAP. The blue line is the distribution of FRBs and the shadow region is the uncertainties of the redshifts. Using the time delay $\tau=5.50 \mathrm{Gyr}, \gamma=2.07$ and the metallicity $Z=0.52 Z_{\odot}$, we give the best fitting as the red line.

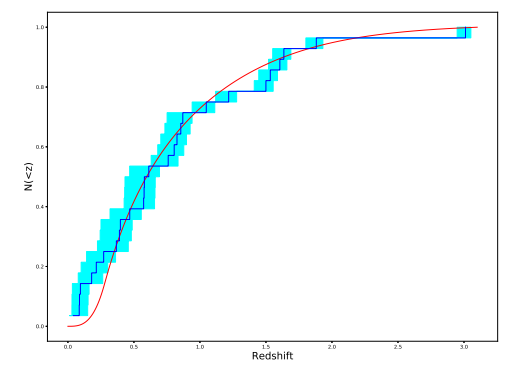

Figure 3. The cumulative distribution of 28 FRBs observed by Parkes. The blue line is the observed distribution of FRBs and the shadow regions is the uncertainties of the redshifts. The bestfitting is given by the red line with $\gamma=2.37$ and $Z=0.52 Z_{\odot}$

This paper has been typeset from a $\mathrm{T}_{\mathrm{E}} \mathrm{X} / \mathrm{LAT}_{\mathrm{E}} \mathrm{X}$ file prepared by the author.

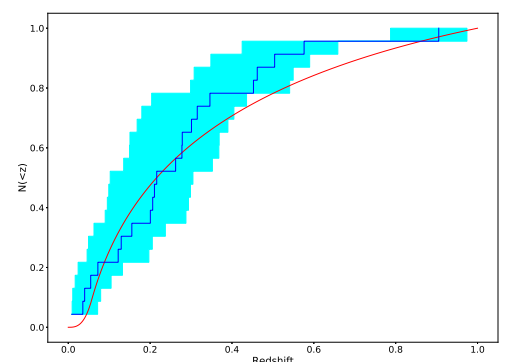

Figure 4. The cumulative distribution of 23 FRBs observed by ASKAP. The blue line is the observed distribution of FRBs and the shadow region is the uncertainties of the redshifts. The bestfitting is given by the red line with $\gamma=2.40$ and $Z=0.52 Z_{\odot}$. 
Parkes (with time delay) ASKAP(with time delay) Parkes (without time delay) ASKAP (without time delay)

\begin{tabular}{ccccc}
\hline$\tau(\mathrm{Gyr})$ & $2.77_{-1.90}^{+2.86}$ & $5.50_{-3.62}^{+3.01}$ & & \\
\hline$\gamma$ & $1.63_{-0.25}^{+0.32}$ & $2.07_{-0.14}^{+0.14}$ & $2.37_{-0.16}^{+0.12}$ & $2.40_{-0.08}^{+0.08}$ \\
\hline$Z\left(Z_{\odot}\right)$ & $0.46_{-0.31}^{+0.35}$ & $0.52_{-0.34}^{+0.32}$ & $0.52_{-0.34}^{+0.34}$ & $0.52_{-0.34}^{+0.32}$ \\
\hline $\mathrm{p}$-value & 0.41 & 0.92 & 0.55 & 0.78 \\
\hline
\end{tabular}

Table 1. Best-fitting Results. 